\title{
SIEGEL MODULAR FORMS OF GENUS 2 ATTACHED TO ELLIPTIC CURVES
}

\author{
Dinakar RAMakrishnan AND Freydoon Shahidi
}

\section{Introduction}

The object of this article is to construct certain classes of arithmetically significant, holomorphic Siegel cusp forms F of genus 2, which are neither of Saito-Kurokawa type, in which case the degree 4 spinor $L$-function $L(s, F)$ is divisible by an abelian $L$-function, nor of Yoshida type, in which case $L(s, F)$ is a product of $L$-series of a pair of elliptic cusp forms. In other words, for each $F$ in the class of holomorphic Siegel cusp forms we construct below, which includes those of scalar weight $\geq 3$, the cuspidal automorphic representation $\pi$ of $\operatorname{GSp}(4, \mathbb{A})$ generated by $F$ is neither $C A P$, short for Cuspidal Associated to a Parabolic, nor endoscopic, i.e., one arising as the transfer of a cusp form on $\mathrm{GL}(2) \times \mathrm{GL}(2)$, and consequently, $L(s, F)$ is not a product of $L$-series of smaller degrees.

A key example of the first kind of Siegel modular forms we construct is furnished by the following result, which will be used in [34]:

Theorem A Let $E$ be an non-CM elliptic curve over $\mathbb{Q}$ of conductor $N$. Then there exists a holomorphic, Hecke eigen-cusp form $F$ of weight 3 acting on the 3-dimensional Siegel upper half space $\mathfrak{H}_{2}$, such that

$$
L(s, F)=L\left(s, \operatorname{sym}^{3}(E)\right) .
$$

Moreover, $F$ has the correct level, i.e., what is given by the conductor $M$ of the Galois representation on the symmetric cube of the Tate module $T_{\ell}(E)$ of $E$.

Such an $F$ is well known to generate a non-zero $(3,0)$-cohomology class on a smooth toroidal compactification $\tilde{S}_{\Gamma}$ of the quotient of the genus 2 Siegel upper half space $\mathfrak{H}_{2}$ by a congruence subgroup

$$
\Gamma \subset \operatorname{Sp}(4, \mathbb{Z}):=\left\{\gamma \in \operatorname{SL}(4, \mathbb{Z}) \mid{ }^{t} \gamma\left(\begin{array}{cc}
0 & I \\
-I & 0
\end{array}\right) \gamma=\left(\begin{array}{cc}
0 & I \\
-I & 0
\end{array}\right)\right\} .
$$

When we say that $\Gamma$ is of level $M$, we mean that it contains the principal congruence subgroup of level $M$ :

$$
\Gamma^{(2)}(M):=\operatorname{Ker}(\operatorname{Sp}(4, \mathbb{Z}) \rightarrow \operatorname{Sp}(4, \mathbb{Z} / M \mathbb{Z})),
$$

but without containing $\Gamma^{(2)}\left(M^{\prime}\right)$ for any $M^{\prime}<M$. There is as of yet no satisfactory theory of Siegel newforms except when $M$ is square-free ( [41]).

Received by the editors August 31, 2006. 
Furthermore, for any $N, L\left(s, \operatorname{sym}^{3}(E)\right)=\prod_{p} L_{p}\left(s, \operatorname{sym}^{3}(E)\right)$ is the symmetric cube $L$-function of $E$. To be precise, if $L(s, E)=\prod_{p}\left(1-a_{p} p^{-s}+p^{1-2 s}\right)^{-1}$ is the $L$-series of $E$, then for any prime $p$ not dividing $N, a_{p}=p+1-\left|E\left(\mathbb{F}_{p}\right)\right|$ equals $\alpha_{p}+\beta_{p}$ with $\alpha_{p} \beta_{p}=p$. The Euler factors of $L\left(s, \operatorname{sym}^{3}(E)\right)$ are given (for such unramified $p$ ) by

$$
L_{p}\left(s, \operatorname{sym}^{3}(E)\right)=\left[\left(1-\alpha_{p}^{3} p^{-s}\right)\left(1-\alpha_{p}^{2} \beta_{p} p^{-s}\right)\left(1-\alpha_{p} \beta_{p}^{2} p^{-s}\right)\left(1-\beta_{p}^{3} p^{-s}\right)\right]^{-1} .
$$

Of course we know now, thanks to the deep works of Wiles, Taylor, Diamond, Conrad and Breuil ( $[55],[50],[6])$, that every $E / \mathbb{Q}$ is modular and attached to a holomorphic newform $f$ on the upper half plane $\mathfrak{H}_{1} \subset \mathbb{C}$ of weight 2 , level $N$ and trivial character.

Our result extends to give the existence of a holomorphic Siegel cusp form $F$ corresponding to the symmetric cube of any newform $f$ of even weight $k \geq 2$ and trivial character (see Theorem $A^{\prime}$ in Section 1). In particular, every newform $f$ of weight $2 k$ for $\mathrm{SL}(2, \mathbb{Z})$, for instance the ubiquitous $\Delta$ function of weight 12 , gives rise to a vector valued Siegel modular cusp form $F$ of genus 2 for $\operatorname{Sp}(4, \mathbb{Z})$. Such an $F$ can be viewed as a section of a holomorphic vector bundle $\mathcal{V}$ of rank $2 k-1$ over $\tilde{S}_{\mathrm{Sp}(4, \mathbb{Z})}$ defined by the representation $\operatorname{det}^{2 k+1} \otimes \mathrm{sym}^{2 k-2}$ of the maximal compact subgroup $\mathrm{U}(2)$ of $\operatorname{Sp}(4, \mathbb{R})$. Earlier, some numerical evidence for the existence of $F$ was given by T. Ibukiyama in [20], and our result in the level 1 case settles the conjecture therein. The starting point for the proof of our Theorem A is the symmetric cube transfer, achieved in [29] by the second author and H. Kim, of automorphic forms from GL(2) to GL(4).

A typical example of the second kind of holomorphic Siegel modular forms we construct is given by the following:

Theorem B Let $E$ be an elliptic curve over $\mathbb{Q}$ and $K$ an imaginary quadratic field with non-trivial automorphism $\theta$ which does not embed into $\operatorname{End}_{\overline{\mathbb{Q}}}(E) \otimes \mathbb{Q}$. Pick any algebraic Hecke character $\chi$ of $K$ of even non-zero weight, with associated $\ell$-adic character $\chi_{\ell}$, which is anti-cyclotomic, i.e., satisfies $\chi^{\theta}=\chi^{-1}$. Then there exists a holomorphic, Siegel modular, Hecke eigen-cusp form F, whose level is the conductor of the $G a l(\overline{\mathbb{Q}} / \mathbb{Q})$-representation on $T_{\ell}(E) \otimes \operatorname{Ind}_{K}^{\mathbb{Q}}\left(\chi_{\ell}\right)$, such that, as Euler products over $\mathbb{Q}$,

$$
L(s, F)=L\left(s, E_{K}, \chi\right) .
$$

Here $L\left(s, E_{K}, \chi\right)$ denotes the $\chi$-twisted $L$-function of $E$ over $K$, which defines a degree 2 Euler product over $K$. Viewed as a degree 4 Euler product over $\mathbb{Q}$, it identifies with $L\left(s, T_{\ell}(E) \otimes \operatorname{Ind}_{K}^{\mathbb{Q}}\left(\chi_{\ell}\right)\right)$. Here $\operatorname{Ind}_{K}^{\mathbb{Q}}$ is short-hand for the induction of a $\operatorname{Gal}(\overline{\mathbb{Q}} / K)$-module to one of $\operatorname{Gal}(\overline{\mathbb{Q}} / \mathbb{Q})$. If $N$ is prime to the conductor $N^{\prime}$ of $\operatorname{Ind}_{K}^{\mathbb{Q}}\left(\chi_{\ell}\right)$, which equals the norm (to $\mathbb{Q}$ ) of the conductor of $\chi$ times the absolute value of the discriminant $D$ of $K$, then the level of $F$ is given by $M=N^{2} N^{\prime 2}$.

It was A. Wiles who brought the problem of proving Theorem B to the attention of the first author a few years ago, and our work on this article began with his question. It is important to note that there is no intersection of our result with the elegant theorem of R.L. Taylor, M. Harris and D. Soudry ( [16]), associating holomorphic Siegel modular forms to certain cusp forms on $\mathrm{GL}(2) / K$. Indeed, for their work to 
apply in our setting, we would need $\chi^{2}$ to be fixed by $\theta$, forcing an anti-cyclotomic $\chi$ to have order 4 , hence of weight zero. We expect Theorem B to hold for $\chi$ of any weight, as long as it is not fixed by $\theta$, and a proof will presumably come out of a blending, and strengthening, of the results of Arthur ([1]) and Whitehouse ( [54]).

In Section 2 we establish an extension (see Theorem $\mathrm{B}^{\prime}$ ) for newforms $\varphi$ of weight $k \geq 2$ relative to anti-cyclotomic characters $\chi$ of any imaginary quadratic field $K$ of weight $w \neq 0$ of the same parity (as $k$ ). The resulting genus 2 Siegel cusp form $F$ turns out to be scalar-valued of (Siegel) weight $k$ exactly when $k=w+2$.

These two kinds of forms can be interpreted, thanks to some recent results such as [29], [28], as special cases of the following general construction. First we need some preliminaries. An isobaric automorphic representation $\pi$ of $\mathrm{GL}_{n}(\mathbb{A})$ is said to be algebraic ( [10]) if the restriction of the associated $n$-dimensional representation $\sigma\left(\pi_{\infty} \otimes|\cdot|^{(1-n) / 2}\right)$ of the real Weil group $W_{\mathbb{R}}$ to its subgroup $\mathbb{C}^{*}$ is of the form $\oplus_{j=1}^{n} \chi_{j}$, with each $\chi_{j}$ algebraic, i.e., of the form $z \rightarrow z^{p_{j}} \bar{z}^{q_{j}}$ with $p_{j}, q_{j} \in \mathbb{Z}$. One says that $\pi$ is regular iff $\left.\sigma\left(\pi_{\infty}\right)\right|_{\mathbb{C}^{*}}$ is a direct sum of characters $\chi_{j}$, each occurring with multiplicity one.

Given a prime $\ell$ and a finite set $S$ of places containing $\ell$ and $\infty$, we will say that a regular, algebraic cusp form $\pi$ on $\operatorname{GL}(n) / \mathbb{Q}$ is of $\ell$-adic Galois type relative to $S$ if there is an $n$-dimensional semisimple $\ell$-adic representation $\rho_{\ell}$ of $\mathrm{Gal}(\overline{\mathbb{Q}} / \mathbb{Q})$ such that for a finite set of places $S$ containing the ramified primes and $\ell$,

$$
L^{S}(s-w / 2, \pi)=L^{S}\left(s, \rho_{\ell}\right),
$$

for a suitable "weight" $w$. For $n=2$ and $\pi$ defined by a holomorphic newform of weight $k \geq 2$, this is well known by [13]. For any $n$, if $\pi$ is selfdual, regular, and has a discrete series component at a prime $r$, say, one knows by Clozel [11]) that it is of $\ell$-adic Galois type.

If $\Pi$ is a cuspidal automorphic representation of $\operatorname{GSp}(4, \mathbb{A})$, we will say that it has level $M \geq 0$ iff its finite component $\Pi_{f}$ has a vector invariant under a compact open subgroup $K$ of $\operatorname{GSp}(4, \hat{\mathbb{Z}})$ which contains the principal congruence subgroup $K(M)$ of level $M$, but does not contain $K\left(M^{\prime}\right)$ for any non-negative $M^{\prime}<M$; the dimension of such invariant vectors need not be 1 . The intersection of $K(M)$ with $\operatorname{Sp}(4, \mathbb{Z})$ is $\Gamma^{(2)}(M)$.

Theorem $\mathbf{C}$ Let $\pi$ be a cuspidal automorphic representation of $G L_{4}(\mathbb{A})$ of conductor $M$, whose exterior square $L$-function has a pole, i.e., one has

$$
-\operatorname{ord}_{s=1} L\left(s, \pi ; \Lambda^{2}\right)=1 .
$$

Then the following hold:

(a) There exists a cuspidal automorphic representation $\Pi=\Pi_{\infty} \otimes \Pi_{f}$ of $G S p_{4}(\mathbb{A})$ (of trivial central character), which is not CAP or endoscopic, such that

$$
L\left(s, \Pi ; r_{5}\right) \zeta(s)=L\left(s, \pi ; \Lambda^{2}\right),
$$

where the first $L$-function on the left is the degree 5 L-function of $\Pi$; moreover, $\Pi_{f}$ has a non-zero vector invariant under a compact open subgroup $K^{M}=$ 


$$
\begin{aligned}
& \prod_{p} K_{p}^{M} \text { of } \operatorname{GSp}\left(4, \mathbb{A}_{f}\right) \text {, of level } M \text {, and if } p \text { divides } M \text { at most once, we can } \\
& \text { take } \\
& K_{p}^{M}=K_{p, I}(M):=\left\{k_{p} \in \operatorname{GSp}\left(4, \mathbb{Z}_{p}\right) \mid k_{p} \equiv\left(\begin{array}{llll}
* & 0 & * & * \\
* & * & * & * \\
0 & 0 & * & * \\
0 & 0 & 0 & *
\end{array}\right) \quad\left(\bmod p^{v_{p}(M)}\right)\right\} .
\end{aligned}
$$

(b) Suppose $\pi$ is in addition regular and algebraic. Then there is a cusp form $\Pi^{h}$ on $G S p(4) / \mathbb{Q}$ with $\Pi_{\infty}$ in the holomorphic discrete series, with $\Pi^{h}$ not $C A P$ or endoscopic, such that the identity of (i) holds with $\Pi^{h}$ in the place of $\Pi$.

(c) If $\pi$ is as in (b) and is moreover of $\ell$-adic Galois type for some $(\ell, S)$, then

$$
L\left(s, \Pi^{h} ; r_{4}\right)=L(s, \pi),
$$

where the L-function on the left is the degree 4 (spin) L-function of $\Pi^{h}$.

For the basic properties of the exterior square $L$-function, see [23] and [7]. It is useful to note that when $L\left(s, \pi ; \Lambda^{2}\right)$ has a pole at $s=1, \pi$ is necessarily selfdual. This is so because

$$
L(s, \pi \times \pi)=L\left(s, \pi ; \Lambda^{2}\right) L\left(s, \pi ; \operatorname{sym}^{2}\right),
$$

where the symmetric square $L$-function ([8]) on the right has no zero at $s=1$ by [45], the reason being that $\pi$ is generic, being cuspidal on $\operatorname{GL}(n)$; moreover, the RankinSelberg $L$-function on the left has a pole at $s=1$ iff $\pi \simeq \pi^{\vee}$.

In view of the result of Clozel alluded to just before this Theorem, we see that the stronger identity (ii) (of Theorem C) holds for any regular, algebraic $\pi$ with a discrete series component at a prime with its exterior square $L$-function having a pole at $s=1$.

We make use of a number of beautiful results due to others, such as the works of Taylor, Clozel, Rallis, Cogdell, Piatetskii-Shapiro, Ginzburg, Harris, Jiang, Soudry, Laumon, and Weissauer, as well as the joint works of the second author with Kim.

The proofs in this article would have been much shorter had an unpublished result of Jacquet, Piatetski-Shapiro and Shalika, concerning the descent of automorphic forms of symplectic type from GL(4) to $\operatorname{GSp}(4)$, been available. To make up for it, we use a detour and go via $\mathrm{SO}(5)$, which makes us lose some information (due to the difference between the degree 4 and degree $5 L$-functions), recovered later by an argument involving Galois representations. In the last Section we speculate on how one could avoid the arithmetic argument if one knew multiplicity one for $\operatorname{Sp}(4)$; it will strengthen Theorem C.

\section{Why Theorem $\mathrm{C}$ implies Theorem A}

Let $E$ be an elliptic curve over $\mathbb{Q}$ of conductor $N$. Then by [6], there is a holomorphic newform $h$ of weight 2 , level $N$ and trivial character such that $L(s, E)=L(s, h)$.

Let $\mathbb{A}=\mathbb{R} \times \mathbb{A}_{f}$ be the adele ring of $\mathbb{Q}$, with $\mathbb{A}_{f}=\hat{\mathbb{Z}} \otimes \mathbb{Q}$, where $\hat{\mathbb{Z}}:=\lim _{n} \mathbb{Z} / n \mathbb{Z} \simeq$ $\prod_{p} \mathbb{Z}_{p}$. Then it is well known that such an $h$ generates a cuspidal automorphic representation $\eta=\eta_{\infty} \otimes \eta_{f}$ of $\mathrm{GL}_{2}(\mathbb{A})=\mathrm{GL}(2, \mathbb{R}) \times \mathrm{GL}\left(2, \mathbb{A}_{f}\right)$ of trivial central character 
and conductor $N$ such that the archimedean component $\eta_{\infty}$ is in the lowest discrete series $\mathcal{D}_{2}$. The associated representation of the real Weil group $W_{\mathbb{R}}:=\mathbb{C}^{*} \cup j \mathbb{C}^{*}$, $j^{2}=-1, j z j^{-1}=\bar{z}$ (for all $z \in \mathbb{C}^{*}$ ), is given, in the unitary form, by the following:

$$
\sigma_{\infty}(\eta) \simeq \operatorname{Ind}_{\mathbb{C}^{*}}^{W_{\mathbb{R}}}\left(\frac{z}{|z|}\right)
$$

Consequently, its restriction to $\mathbb{C}^{*}$ is given by the direct sum of characters in the infinity type:

$$
p_{\infty}=\left\{\frac{z}{|z|}, \frac{\bar{z}}{|z|}\right\}
$$

This is so because $h$ contributes to the $H^{1}$ of the modular curve, which has Hodge weight 1 .

Note that $E$ has complex multiplication (CM) by (an order in) an imaginary quadratic field $K$ iff $\eta$ is automorphically induced by a Hecke character of $K$, necessarily of weight 1 . Since we assume that $E$ has no $\mathrm{CM}, \eta$ is not dihedral.

Here is a gentle strengthening of Theorem A:

Theorem $\mathbf{A}^{\prime}$ Let $\eta$ be a cuspidal automorphic representation of $G L(2, \mathbb{A})$ defined by a holomorphic, non-CM newform $\varphi$ of even weight $k \geq 2$, level $N$ and trivial character. Then there exists a cuspidal automorphic representation $\Pi$ of $G \operatorname{Sp}(4, \mathbb{A})$ of trivial character, which is unramified at any prime $p$ not dividing $N$, such that

(a) $\Pi_{\infty}$ is in the holomorphic discrete series, with its parameter being the symmetric cube of that of $\sigma_{\infty}$; and

$$
L(s, \Pi)=L\left(s, \eta, \mathrm{sym}^{3}\right) .
$$

Moreover, $\Pi$ has a non-zero vector invariant under a compact open subgroup $K$ of $G S p\left(4, \mathbb{A}_{f}\right)$ of level equal to the conductor $M$ of the symmetric cube of the Galois representation attached to $\varphi$.

If $\eta_{p}$ is determined at any unramified prime $p$ by an unordered pair $\left\{\alpha_{p}, \beta_{p}\right\}$, then the $L$-function on the right of (b) has an Euler product whose $p$-factors outside $N$ have the inverse roots:

$$
\left\{\alpha_{p}^{3}, \alpha_{p}^{2} \beta_{p}, \alpha_{p} \beta_{p}^{2}, \beta_{p}^{3}\right\}
$$

In fact, at any prime $p$, the local factors such as $L\left(s, \eta_{p} ; \operatorname{sym}^{3}\right)$ can be defined as the local factors of the associated representations $\sigma\left(\eta_{p}\right)$ of the extended Weil group $W_{\mathbb{Q}_{p}}^{\prime}$, which is possible thanks to the local Langlands correspondence for $\mathrm{GL}(n)$ ( [17], [18]).

Now we show why Theorem $\mathrm{C}$ implies Theorem $A^{\prime}$ (and hence Theorem $A$ ). A theorem of the second author with H. Kim ( $[29]$ ), asserts that there is an isobaric automorphic representation $\operatorname{sym}^{3}(\eta)$ of $\mathrm{GL}_{4}(\mathbb{A})$ such that

$$
L\left(s, \operatorname{sym}^{3}(\eta)\right)=L\left(s, \eta ; \operatorname{sym}^{3}\right),
$$

and moreover, the archimedean parameter of $\operatorname{sym}^{3}(\eta)$ is the symmetric cube of that of $\eta$.

Put

$$
\pi=\operatorname{sym}^{3}(\eta)
$$


Then, as $\eta$ is defined by a holomorphic newform of weight $k$, its archimedean parameter is, in the unitary form,

$$
\left\{\left(\frac{z}{|z|}\right)^{k-1},\left(\frac{\bar{z}}{|z|}\right)^{k-1}\right\}
$$

It follows that the parameter of $\pi_{\infty}$ is

$$
\left\{\left(\frac{z}{|z|}\right)^{3(k-1)},\left(\frac{z}{|z|}\right)^{k-1},\left(\frac{\bar{z}}{|z|}\right)^{k-1},\left(\frac{\bar{z}}{|z|}\right)^{3(k-1)}\right\} .
$$

So $\pi$ is algebraic and regular. Moreover, since the central character $\omega$ of $\eta$ is trivial, it is selfdual (as $\eta^{\vee} \simeq \eta \otimes \omega^{-1}$ ), and so is $\pi=\operatorname{sym}^{3}(\eta)$.

Next, since $\eta$ is generated by a holomorphic newform of weight $2 k \geq 2$ and level $N$, we know by Deligne the existence, for any prime $\ell$, of an (irreducible) $\ell$-adic representation $\tau_{\ell}$ of $\operatorname{Gal}(\overline{\mathbb{Q}} / \mathbb{Q})$ ) such that for all primes $p$ not dividing $N \ell$, the following identity of local factors holds:

$$
L_{p}(s-(k-1) / 2, \eta)=L_{p}\left(s, \tau_{\ell}\right) .
$$

It follows that $\operatorname{sym}^{3}\left(\tau_{\ell}\right)$ is associated to $\pi$. Furthermore, one knows (cf. [9]) that the conductors of $\eta$ and $\tau_{\ell}$ coincide. When this is coupled with the fact that the symmetric cube transfer $\eta \rightarrow \operatorname{sym}^{3}(\eta)$ is functorial at every prime, one deduces the equality of the conductors of $\operatorname{sym}^{3}(\eta)$ and $\operatorname{sym}^{3}\left(\tau_{\ell}\right)$.

The automorphic representation $\pi$ is cuspidal, as one sees from Remark 5.9 and Proposition 5.11 of [47]. Here are two other proofs of the same assertion, both using more the associated $\ell$-adic representation. The starting point is again that since $\eta$ is non-dihedral (by hypothesis), [28] implies that $\pi$ is cuspidal unless the symmetric square $\operatorname{sym}^{2}(\eta)$ of $\eta$ is monomial, i.e., admits a non-trivial self-twist. But in that case, by the Tchebotarev density theorem, $\operatorname{sym}^{2}\left(\tau_{\ell}\right)$ would also admit a non-trivial selftwist, making its restriction to an open subgroup $\mathrm{Gal}(\overline{\mathbb{Q}} / F)$ reducible, with $F$ cyclic of degree 3. But this would contradict a theorem of Ribet ( $[38])$, asserting that the Zariski closure of the image of $\operatorname{Gal}(\overline{\mathbb{Q}} / \mathbb{Q})$ in $\mathrm{GL}\left(2, \mathbb{Q}_{\ell}\right)$ (under $\left.\tau_{\ell}\right)$ contains $\operatorname{SL}\left(2, \mathbb{Q}_{\ell}\right)$. Alternatively, we see that the base change $([2])$ of $\operatorname{sym}^{2}(\pi)$ to the totally real $F$ is not cuspidal, while the associated Galois representation $\operatorname{sym}^{2}\left(\tau_{\ell}\right)$ is irreducible when restricted to $\operatorname{Gal}(\overline{\mathbb{Q}} / F)$, contradicting the main result (for $n=3$ ) of [37].

To satisfy the hypotheses of Theorem $C$, it remains only to show that $\pi$ is of symplectic type, i.e., that the exterior square $L$-function $L\left(s, \pi ; \Lambda^{2}\right)$ has a pole at $s=1$. For this we appeal to the (well known) identity

$$
L\left(s, \pi ; \Lambda^{2}\right)=L\left(s, \eta ; \operatorname{sym}^{4}\right) \zeta(s),
$$

which is immediate at the unramified primes $p$. It is known that the symmetric 4-th power $L$-function of $\eta$ has no zero at $s=1$. (One even knows by H. Kim ( [27]) that there is a corresponding automorphic form $\operatorname{sym}^{4}(\eta)$ on $\mathrm{GL}(5) / \mathbb{Q}$, but we do not need to appeal to it at this point.) Thus the pole of $\zeta(s)$ at $s=1$ induces one of $L\left(s, \pi ; \Lambda^{2}\right)$, and Theorem $C$ can be applied with $\pi=\operatorname{sym}^{3}(\eta)$. One gets the truth of Theorems $A$ and $A^{\prime}$. 


\section{Why Theorem C implies Theorem B}

Let $E$ be an elliptic curve over $\mathbb{Q}$, with associated cusp form $\eta$ on $\operatorname{GL}(2) / \mathbb{Q}$, and let $K$ be an imaginary quadratic field (with non-trivial automorphism $\theta$ ), which does not lie in the $\mathbb{Q}$-endomorphism algebra of $E$. Then $\eta$ is not dihedral relative to $K$, or in other words, the base change $\eta_{K}$ (of $\eta$ to $\mathrm{GL}(2) / K$ ) remains cuspidal. Pick any anticyclotomic Hecke character $\chi$ of $K$ of of even weight $w \neq 0$. We will use $\chi$ to again denote the associated idele class character, and use $\chi_{\ell}$ to denote the corresponding $\ell$-adic character ( $[44])$ of $\operatorname{Gal}(\bar{K} / K)$. One has

$$
L\left(s, E_{K}, \chi\right)=L\left(s, \eta_{K} \otimes \chi\right)=L\left(s, \eta \times I_{K}^{\mathbb{Q}}(\chi)\right),
$$

where $I_{K}^{\mathbb{Q}}(\chi)$ denotes the (automorphically induced) cusp form on $\operatorname{GL}(2) / \mathbb{Q}$ attached to $\chi$, defined by a holomorphic newform of weight $w+1$, constructed in this case by Hecke. Since $\chi$ has a non-zero weight, it cannot be fixed by $\theta$ and so $I_{K}^{\mathbb{Q}}(\chi)$ is indeed cuspidal. The $L$-function on the right of (2.1) is the Rankin-Selberg $L$-function attached to the pair $\left(\eta, I_{K}^{\mathbb{Q}}(\chi)\right)$.

Here is a gentle strengthening of Theorem B:

Theorem $\mathbf{B}^{\prime}$ Let $K$ be an imaginary quadratic field of discriminant $D<0$ and let $\delta=\delta_{K / \mathbb{Q}}$ denote the quadratic Dirichlet character of $\mathbb{Q}$ associated to $K$. Consider any cuspidal, automorphic representation $\eta$ of $G L(2, \mathbb{A})$ defined by a holomorphic newform of weight $k \geq 2$, level $N$ and character $\omega$, which is trivial if $k$ is even and $\delta$ if $k$ is odd. Let $\chi$ be an anti-cyclotomic Hecke character of weight $w \neq 0$, such that $w$ and $k$ have same parity. Then there exists a cuspidal automorphic representation $\Pi$ of $G S p(4, \mathbb{A})$ of trivial central character, which is unramified at any prime $p$ not dividing $N D$, such that

(a) $\Pi_{\infty}$ is in the holomorphic discrete series, with its parameter being the tensor product of those of $\eta_{\infty}$ and $I_{K}^{\mathbb{Q}}(\chi)_{\infty} ;$ and

(b) As Euler products over $\mathbb{Q}$,

$$
L(s, \Pi)=L\left(s, \eta_{K} \otimes \chi\right) .
$$

(c) $\Pi$ defines a scalar-valued Siegel modular cusp form $F$ when $k=w+2$.

Now we show why Theorem $\mathrm{C}$ implies Theorem $B^{\prime}$ (and hence Theorem $B$ ). As $\chi$ is anti-cyclotomic, it can be fixed by $\theta$ iff it is quadratic, which is impossible as it has non-zero weight. Hence $\eta_{K} \otimes \chi$ can be $\theta$-invariant iff it admits a non-trivial self-twist by $\chi^{2}\left(=\chi / \chi^{\theta}\right)$, which is again impossible because any such self-twisting character has to be at most quadratic. Thus $\eta_{K} \otimes \chi \chi\left(\eta_{K} \otimes \chi\right)^{\theta}$, and so by the base change theory of Arthur and Clozel [2] (see also [19]), it has a cuspidal automorphic induction

$$
\pi:=I_{K}^{\mathbb{Q}}\left(\eta_{K} \otimes \chi\right)
$$

to $\mathrm{GL}(4) / \mathbb{Q}$. Alternately, one has a cuspidal automorphic representation $\eta \otimes I_{K}^{\mathbb{Q}}(\chi)$ of $\mathrm{GL}_{4}(\mathbb{A})$ (cf. [35]), such that

$$
L\left(s, \eta \otimes I_{K}^{\mathbb{Q}}(\chi)\right)=L\left(s, \eta \times I_{K}^{\mathbb{Q}}(\chi)\right)
$$

It follows by the strong multiplicity one theorem that

$$
\pi \simeq \eta \otimes I_{K}^{\mathbb{Q}}(\chi) .
$$


Unraveling this isomorphism, we see (using (1.6)) that the archimedean parameter of $\pi$ is given by

$$
\left\{\left(\frac{z}{|z|}\right)^{(k-1)+w},\left(\frac{z}{|z|}\right)^{(k-1)-w},\left(\frac{\bar{z}}{|z|}\right)^{(k-1)-w},\left(\frac{\bar{z}}{|z|}\right)^{(k-1)+w}\right\} .
$$

Since $w$ is non-zero and has the same parity as $k, k-1 \pm w$ cannot be zero and $k-1+w \neq k-1-w$. In other words, $\pi$ is regular and algebraic.

We will now show that $\pi$ is of symplectic type, which will imply in particular that it is selfdual. For this we appeal to the identity

$$
L\left(s, \pi ; \Lambda^{2}\right)=L\left(s, \operatorname{sym}^{2}(\eta) \otimes \chi_{0} \delta\right) L\left(s, \omega \otimes\left(I_{K}^{\mathbb{Q}}\left(\chi^{2}\right)\right) L\left(s, \omega \chi_{0}\right),\right.
$$

where we have used the fact that the central character of $I_{K}^{\mathbb{Q}}(\chi)$ is $\delta$ times the restriction $\chi_{0}$ of $\chi$ to (the idele classes of) $\mathbb{Q}$, and that

$$
\operatorname{sym}^{2}\left(I_{K}^{\mathbb{Q}}(\chi)\right) \simeq I_{K}^{\mathbb{Q}}\left(\chi^{2}\right) \boxplus \chi_{0} .
$$

Now since $\chi$ is anti-cyclotomic,

$$
\chi_{0} \circ N_{K / \mathbb{Q}}=\chi \chi^{\theta}=1,
$$

implying that $\chi_{0}$ is $\delta^{w}$. Since $w$ and $k$ have the same parity, as does $\omega$, we deduce that $\omega \chi_{0}$ is trivial. Hence by $(2.6), L\left(s, \pi ; \Lambda^{2}\right)$ is $\zeta(s)$ times a product of known $L$-functions which do not have a zero at $s=1$. Thus $\pi$ is symplectic.

To finish proving that Theorem $C$ implies Theorems $B$ and $B^{\prime}$, it remains only to check that the holomorphic Siegel cusp form $F$ which $\pi$ defines is scalar-valued of weight $k$ when $k=w+2$. But the archimedean parameter of a Siegel modular form of scalar weight $k \geq 2$ is known to be of the form

$$
\left\{\left(\frac{z}{|z|}\right)^{2 k-3}, \frac{z}{|z|}, \frac{\bar{z}}{|z|},\left(\frac{\bar{z}}{|z|}\right)^{2 k-3}\right\}
$$

Now we are done by comparing this with (2.5).

\section{Transfer to $\operatorname{GSp}(4)$ via $\mathbf{S O}(5)$}

Let $\pi$ be as in Theorem $C$. Since by hypothesis, $L\left(s, \pi ; \Lambda^{2}\right)$ has a pole at $s=1$, where $S$ is a finite set of places containing the ramified and archimedean primes, its global parameter takes values in $\operatorname{Sp}(4, \mathbb{C})$, which is the $L$-group of $\operatorname{SO}(5)$. By the descent theorem of Ginzburg, Rallis and Soudry ( [15], [48]), we can find a cuspidal, globally generic automorphic representation $\Pi^{\prime \prime}$ of the $\operatorname{split} \mathrm{SO}(5, \mathbb{A})$ with parameters in $\operatorname{Sp}(4, \mathbb{C})$.

One knows that $\mathrm{SO}(5)$ has a double cover, namely $\mathrm{Spin}(5)$, which is isomorphic to $\mathrm{Sp}(4)$. Using this we lift $\Pi^{\prime \prime}$ to a cuspidal, globally generic automorphic representation $\Pi^{\prime}$ of $\operatorname{Sp}(4, \mathbb{A})$ with trivial central character.

At any prime $p$ where $\Pi^{\prime}$ is unramified, the parameter of its $p$-component is given by the diagonal element $\Lambda^{2}\left(A_{\pi_{p}}\right)-\operatorname{diag}(0,0,0,0,0,1)$, where $A_{\pi_{p}}$ is the Hecke parameter for $\pi_{p}$, viewed as an element in $\mathrm{SO}(5, C)$. Let $r$ be the standard (5-dimensional) representation of the dual group of $\mathrm{Sp}(4)$, which is $\mathrm{SO}(5, \mathbb{C})$. 
Proposition 3.1 With $\pi, \Pi^{\prime}$ as above, we have

(a)

$$
\begin{gathered}
L\left(s, \Pi^{\prime} ; r\right) \zeta(s)=L\left(s, \pi ; \Lambda^{2}\right), \\
\sigma_{\infty}\left(\Pi^{\prime}\right) \oplus 1 \simeq \Lambda^{2}\left(\sigma_{\infty}(\pi)\right) .
\end{gathered}
$$

Proof. By the work of the second author with Cogdell, Kim and Piatetski-Shapiro ( $[12]$ ), we can transfer $\Pi^{\prime}$ back to a cusp form $\pi^{\prime}$ on $G L(4) / \mathbb{Q}$ such that the arrow $\Pi_{v}^{\prime} \rightarrow \pi_{v}^{\prime}$ is compatible with the descent of [15], and is functorial at every place $v$ (see [48], [25]). So $\pi^{\prime}$ and $\pi$ are equivalent almost everywhere, hence isomorphic by the strong multiplicity one theorem. So the composition of the parameters of $\Pi^{\prime \prime}$ with the natural embedding $\mathrm{Sp}(4, \mathbb{C}) \hookrightarrow \mathrm{GL}(4, \mathbb{C})$ are the same as the parameters of $\pi$ at the various places $v$. Next denote by $\psi$ the pull-back map on the set of automorphic representations of $\operatorname{SO}(5, \mathbb{A}) \simeq \operatorname{PSp}(4, \mathbb{A})$, taking values in the set of automorphic representations of $\operatorname{Sp}(4, \mathbb{A})$, both modulo equivalence. Then $\psi$ corresponds to the $L$-homomorphism

$$
{ }^{L} \psi: \mathrm{Sp}(4, \mathbb{C}) \rightarrow \mathrm{SO}(5, \mathbb{C}),
$$

defined so that the exterior square of the standard 4-dimensional representation of $\operatorname{Sp}(4, \mathbb{C})$ is isomorphic to $\underline{1} \oplus{ }^{L} \psi$. The assertions of the Proposition now follow.

The next object is to find a generic cuspidal representation of $\operatorname{GSp}(4, \mathbb{A})$ whose restriction to $\operatorname{Sp}(4, \mathbb{A})$ contains $\Pi^{\prime}$. This can be done by imitating what Labesse and Langlands do for SL(2) ( [30]). But we want to refine their construction in such a way that we keep track of what happens at the finite primes in order that we do not introduce new ramification. Here is what we do

First note that in the descent construction $\pi \rightarrow \Pi^{\prime}$ of Ginzburg, Rallis and Soudry, the authors first define an Eisenstein series $E(g, s ; \pi)$ on $\mathrm{SO}(9, \mathbb{A})$ and take its residue $E_{1}(g: \pi)$. Then they define $\Pi^{\prime}$, which is $\sigma_{2}(\pi)$ in their notation, by restricting to $\mathrm{SO}(5, \mathbb{A})$ a sequence of Fourier coefficients of $E_{1}(g ; \pi)$ along a unipotent subgroup $U(\mathbb{A})$ of $\mathrm{SO}(9, \mathbb{A})$. All this involves a sequence of explicit integrals, and it follows that if $\pi$ has conductor $M$, then $\Pi^{\prime}$ admits a non-zero vector invariant under a principal compact open subgroup $K^{\prime}(M)$ of level $M$. To elaborate, for any $r \geq 1, K^{\prime}(r)$ is the kernel of $\mathrm{SO}(5, \hat{\mathbb{Z}}) \rightarrow \mathrm{SO}(5, \hat{\mathbb{Z}} / r \hat{\mathbb{Z}})$, and $M$ is the smallest integer $r \geq 0$ such that there is a $K^{\prime}(r)$-fixed vector in $\Pi^{\prime}$. $M$ agrees with the conductor of $\pi$ by the known fact that $\pi$ contains a non-zero vector fixed by the congruence subgroup $K_{0}(M) \subset$ $\mathrm{GL}(4, \hat{\mathbb{Z}})$ of level $M([21])$.

Next we extend $\Pi^{\prime}$ to a representation $\Pi_{1}$ of the group $H:=\operatorname{Sp}(4, \mathbb{A}) Z(\mathbb{A})$, where $Z$ denotes the center of $\operatorname{GSp}(4)$, such that $\Pi_{1}$ is trivial on $Z(\mathbb{A})$. This makes sense because $\Pi^{\prime}$ has trivial central character. Since $\pi$ is unramified outside (the primes dividing) $N, \Pi_{1}$ is also unramified outside $N$. Moreover, the transfer at the ramified primes is still functorial and respects the level. Note that $\operatorname{Sp}(4, \mathbb{A}) Z(\mathbb{A})$ is a normal subgroup of the symplectic similitude group $\operatorname{GSp}(4, \mathbb{A})$ with a countable quotient group. Now induce $\Pi_{1}$ to $\operatorname{GSp}(4, \mathbb{A}$ ), and choose (as follows) a cuspidal automorphic representation $\Pi$ of $\operatorname{GSp}(4, \mathbb{A})$ occurring in the induced representation, which is necessarily globally generic of trivial central character. Let $K(M)$ denote a principal 
congruence subgroup of $\operatorname{GSp}\left(4, \mathbb{A}_{f}\right)$ such that $K_{1}(M):=K(M) \cap \operatorname{Sp}\left(4, \mathbb{A}_{f}\right)$ has image $K^{\prime}(M)$ in $\operatorname{SO}\left(5, \mathbb{A}_{f}\right) \simeq \operatorname{PSp}\left(4, \mathbb{A}_{f}\right)$. Since $\Pi_{1}$ has a fixed vector under $K_{1}(M)$, the induced representation will, by Frobenius reciprocity, have at least one constituent $\Pi$ which will have a vector fixed under $K(M)$, and such a $\Pi$ is what we choose. In particular, $\Pi_{p}$ is unramified whenever $\pi_{p}$ is.

Suppose a prime $p$ divides $M$. Then it is not hard to see that $\Pi$ is not unramified, the reason being that the descent of [15] is compatible with the transfer of [12], which preserves the epsilon factors. So, in particular, if $p$ divides $M$ exactly once, then by appealing to [41], we see that $\Pi_{p}$ must in fact contain a non-zero vector $v$ which is invariant under the Iwahori congruence subgroup $K_{p, I}(M)$ defined in the statement of Theorem C, part (a), in the Introduction, and it is not unramified for a maximal compact subgroup. Again, it is helpful to note that for non-square-free $N$, there is no good candidate for the "conductor", and the space of vectors fixed by $K(M)$ need not be 1-dimensional; for an approach in the paramodular setting, see [39].

For $j=4,5$, let $r_{j}$ denote the $j$-dimensional representation of the dual group of $\operatorname{GSp}(4)$, which can be identified with $\mathrm{GSp}(4, \mathbb{C})$ itself since it is isomorphic to GSpin(5). (We want to be careful because some think of $r_{4}$ as the standard representation, while the others think that it is $r_{5}$.) We get, using part (a) of Prop.3.1,

$$
L\left(s, \Pi ; r_{5}\right) \zeta(s)=L\left(s, \pi ; \Lambda^{2}\right) .
$$

Indeed, as $\Pi$ has trivial central character, the $r_{5}$-parameter of $\Pi_{v}$ is, at any place $v$, the same as the $r$-parameter of $\Pi_{1, v}$.

Proposition 3.4 Let $\Pi$ be as above, being associated to $\pi$. Suppose $\pi$ is regular and algebraic. Then

(a) $\Pi_{\infty}$ is of cohomological type; and

(b) The restriction to $\mathbb{C}^{*}$ of the $r_{4}$-parameter of $\Pi_{\infty}$ is the same as that of $\pi_{\infty}$.

Proof. Since $\pi_{\infty}$ is regular and algebraic, part (a) follows from part (b). Thanks to Proposition 3.1 and the discussion above, we get

$$
\sigma_{\infty}(\Pi) \oplus 1 \simeq \Lambda^{2}\left(\sigma_{\infty}(\pi)\right)
$$

We have

$$
\Lambda^{2} \circ r_{4} \simeq r_{5} \oplus \lambda
$$

where $\lambda$ is the polarization, corresponding to the central character. Consequently,

$$
\Lambda^{2}\left(r_{4}\left(\sigma_{\infty}(\Pi)\right)\right) \simeq \Lambda^{2}\left(\sigma_{\infty}(\pi)\right) .
$$

The parameter of $\pi_{\infty}$ when restricted to $\mathbb{C}^{*}$ will be given by a pair $(a, b)$ with $a, b, a+$ $b \neq 0$ and $a>b$, or more precisely,

$$
\left\{\left(\frac{z}{|z|}\right)^{a},\left(\frac{z}{|z|}\right)^{b},\left(\frac{\bar{z}}{|z|}\right)^{b},\left(\frac{\bar{z}}{|z|}\right)^{a}\right\}
$$

The exterior square parameter will then be determined by the pair $(a+b, a-b)$ (discounting the exponents 0). Similarly, if the $r_{4}$-parameter of $\Pi_{\infty}$ is given by $(c, d)$ with $c>d$, then its exterior square will be determined by $(c+d, c-d)$. It follows that $a=c, b=d$, proving the Proposition. 


\section{Proof of the first two parts of Theorem C}

Let $\Pi$ be attached to a cusp form $\pi$ of symplectic type, as in Section 3 above. Then $\Pi$ is a globally generic, cuspidal automorphic representation of $\operatorname{GSp}(4, \mathbb{A})$ with trivial central character.

We will henceforth denote by $L(s, \Pi)$ the degree four $L$-function of $\Pi$.

Lemma 4.1 $\Pi$ is not a CAP representation, nor is it endoscopic (of Yoshida type), i.e., attached to a pair of cusp forms $\pi_{1}, \pi_{2}$ of $G L(2) / \mathbb{Q}$.

Proof. Since $\Pi$ is generic, it cannot be CAP. Suppose it is endoscopic, associated to a pair of cusp forms $\left(\pi_{1}, \pi_{2}\right)$ of $\mathrm{GL}(2) / \mathbb{Q}$. Then we have

$$
L(s, \Pi)=L\left(s, \pi_{1}\right) L\left(s, \pi_{2}\right) .
$$

In this case the central characters of $\pi_{1}$ and $\pi_{2}$ are trivial, and we have by combining Proposition 3.1 and (4.2),

$$
L\left(s, \pi ; \Lambda^{2}\right)=L\left(s, \pi_{1} \times \pi_{2}\right) \zeta(s)^{2},
$$

where $\pi$ is the cusp form on GL(4)/QQ which descends to $\Pi$. Since the Rankin-Selberg $L\left(s, \pi_{1} \times \pi_{2}\right)$ ( [22], [24], [45]) has no zero at $s=1$, we see that the exterior square $L$-function of $\pi$ has a double pole. It implies, by $(0.1)$, that $L(s, \pi \times \pi)$ has a double pole at $s=1$, which violates the cuspidality of $\pi$. Thus $\Pi$ is not endoscopic.

Part (a) of Theorem $\mathrm{C}$ is now proven, and let us now turn our attention to part (b). From now on, $\pi$ will be regular and algebraic (in addition to being of symplectic type). Thanks to Proposition 3.4, and the regularity of $\pi$, we see that $\Pi_{\infty}$ is squareintegrable. There is a discrete series $L$-packet $\left\{\Pi_{\infty}^{h}, \Pi_{\infty}^{W}\right\}$ of $\operatorname{GSp}(4, \mathbb{R})$ of trivial central character, with $\Pi_{\infty}^{h}$ being holomorphic and $\Pi_{\infty}^{W}$ being of Whittaker type, i.e., generic, such that $\Pi_{\infty}=\Pi_{\infty}^{W}$. It is well known (see [49], p. 293, for example) that such an $L$-parameter contributes to the relative Lie algebra cohomology with coefficients in a finite dimensional representation. Geometrically, this implies that $\Pi$ contributes to the $L^{2}$-cohomology in degree 3 , with coefficients in a suitable local system $V^{a, b}$, of the complex points of the Shimura variety $S_{K}$, defined over $\mathbb{Q}$, attached to a compact open subgroup $K$ of $\operatorname{GSp}\left(4, \mathbb{A}_{f}\right)$. For such a $K, \Pi_{f}^{K}$ is non-zero and is acted on by the Hecke algebra $\mathcal{H}\left(\mathrm{GSp}\left(4, \mathbb{A}_{f}\right), K\right)$ generated by the $K$-double cosets; moreover, it determines $\Pi_{f}$.

Appealing to the proof of Zucker's conjecture due to Looijenga ( [33]) and SaperStern ( [40]), one then obtains, for such a $\Pi$ with $\Pi_{f}^{K} \neq 0$, a class in (the middle intersection cohomology) $X:=I H^{3}\left(S_{K}^{*}(\mathbb{C}), \mathcal{V}^{a, b}\right)$, where $S_{K}^{*}$ is the Baily-Borel-Satake compactification and $\mathcal{V}^{a, b}$ is a locally constant sheaf attached to $V^{a, b}$. By the comparison theorem between Betti (singular) and étale cohomology, $\Pi_{f}$ also contributes, for any prime $\ell$, to the $\ell$-adic cohomology $X_{\ell}:=I H_{\mathrm{et}}^{3}\left(S_{K}^{*} \times \overline{\mathbb{Q}}, \mathcal{V}_{\ell}^{a, b}\right)$, which is pure by Gabber) ( [5]) of weight $a+b-3$, meaning that at any prime $p \neq \ell$ where $X_{\ell}$ is unramified as a Galois representation, the inverse roots of the geometric Frobenius $\operatorname{Fr}_{p}$ are all of absolute value $p^{(a+b-3) / 2}$.

Let $X_{\ell}\left(\Pi_{f}\right)$ denote the isotypic subspace of $X_{\ell} \otimes_{\mathbb{Q}_{\ell}} \overline{\mathbb{Q}}_{\ell}$ defined by $\Pi_{f}$, which is again a $\operatorname{Gal}\left(\overline{\mathbb{Q}} / \mathbb{Q}\right.$ )-module since the action of the Hecke algebra on $X_{\ell}$ (by algebraic 
correspondences) commutes with the Galois action. Its $L$-function is as usual defined by the Euler product

$$
L\left(s, X_{\ell}\left(\Pi_{f}\right)\right)=\prod_{p} \operatorname{det}\left(I-p^{-s} \operatorname{Fr}_{p} \mid X_{\ell}\left(\Pi_{f}\right)^{I_{p}}\right)^{-1},
$$

where $I_{p}$ denotes the inertia group at $p$. The possible Hodge types of the corresponding summand $X_{B}\left(\Pi_{f}\right)$ of the singular cohomology are $(a+b-3,0),(c, d),(d, c),(0, a+b-3)$, with $c+d=a+b-3$. At the primes $p$ where $X_{\ell}\left(\Pi_{f}\right)$ is unramified, i.e., where $I_{p}$ acts trivially, the Frobenius polynomial (defining the Euler factor at $p$ ) has been related to the Hecke polynomial of $\Pi_{p}$ by Weissauer in his article [53], and also by Laumon in [31], [32], who works instead with the cohomology of $S_{K}$ with compact supports. Their beautiful works show, since the cuspidal automorphic representation $\Pi$ is not $\mathrm{CAP}$ or endoscopic, that for some positive integer $m$,

$$
\operatorname{dim} X_{\ell}\left(\Pi_{f}\right)=4 m=2\left(m^{W}\left(\Pi_{f}\right)+m^{h}\left(\Pi_{f}\right)\right),
$$

where $m^{W}\left(\Pi_{f}\right)$, resp. $m^{h}\left(\Pi_{f}\right)$, is the multiplicity of $\Pi_{\infty}^{W} \otimes \Pi_{f}(=\Pi)$, resp. $\Pi_{\infty}^{h} \otimes \Pi_{f}$ in the space of cusp forms on $\operatorname{GSp}(4, \mathbb{A})$. If either of these representations (of discrete series type) occurs in the discrete spectrum of $L^{2}(\operatorname{GSp}(4, \mathbb{Q}) Z(\mathbb{A}) \backslash \operatorname{GSp}(4, \mathbb{A}))$, with $Z$ denoting the center of $\mathrm{GSp}(4)$, then it actually occurs in the cuspidal spectrum. This is because (in degree 3), $X$ lies in cuspidal cohomology (cf. [49], p. 294, for example). Note that the Hodge type $(a+b-3,0)$ occurs in $X_{B}\left(\Pi_{f}\right)$ iff $m^{h}\left(\Pi_{f}\right)$ is non-zero.

In fact, if $S$ denotes the set of ramified and archimedian primes, the results of Weissauer and Laumon (loc. cit.) furnish the following identity: (with $T=S \cup\{\ell\}$ )

$$
L^{T}(s+(3-a-b) / 2, \Pi)^{2\left(m^{W}\left(\Pi_{f}\right)+m^{h}\left(\Pi_{h}\right)\right)}=L^{T}\left(s, X_{\ell}\left(\Pi_{f}\right)\right)^{4} .
$$

Since $\Pi$ is globally generic, we know by a theorem of Jiang and Soudry ( [26]) that $m^{W}\left(\Pi_{f}\right)=1$. Combining these remarks with the results of Weissauer and Laumon above, equation (4.4) in particular, we get

$$
m^{h}\left(\Pi_{f}\right)>0 .
$$

In other words, the holomorphic representation $\Pi^{h}:=\Pi_{\infty}^{h} \otimes \Pi_{f}$ occurs in the space of cusp forms in $L^{2}(\operatorname{GSp}(4, \mathbb{Q}) Z(\mathbb{A}) \backslash \operatorname{GSp}(4, \mathbb{A}))$. Since $\Pi^{h}$ is $L$-equivalent to $\Pi$, it has the same $L$-function. (In fact, the only change is at the archimedean place, where $\Pi_{\infty}^{h}$ and $\Pi_{\infty}$ are in the same discrete series $L$-packet.) Hence by appealing to (3.3) and Proposition 3.4, we get the identity

$$
L\left(s, \Pi^{h} ; r_{5}\right) \zeta(s)=L\left(s, \pi ; \Lambda^{2}\right)
$$

This proves part (b) of Theorem C. Note that (4.5) involves incomplete $L$-functions, but (4.7) equates complete $L$-functions.

Remark 4.8 Exploiting an idea of Taylor ( [49]), one can even deduce from Weissauer's theorem that $m^{h}\left(\Pi_{f}\right)=1$, which yields the identity $L^{T}(s+(3-a-b) / 2, \Pi)=$ $L^{T}\left(s, X_{\ell}\left(\Pi_{f}\right)\right)$. Indeed, thanks to the decomposition theorem ( [5]), $X_{\ell}$ can be seen to be a direct factor of $H_{\mathrm{et}}^{3}\left(\tilde{S}_{K} \times \overline{\mathbb{Q}}, \mathcal{V}_{\ell}^{a, b}\right)$, where $\tilde{S}_{K}$ denotes a smooth toroidal compactification of $S_{K}$, which can be taken to be defined over $\mathbb{Q}$ and such that the complement of $S_{K}$ (in $\tilde{S}_{K}$ ) is a divisor $D$ with normal crossings. It follows, by a theorem of Faltings ( [14], [51]), that $X_{\ell}\left(\Pi_{f}\right)$ is of Hodge-Tate type. Let $\mathcal{G}_{\ell}\left(\Pi_{f}\right)$ denote the Lie algebra of the Zariski closure of the image of Galois in $\operatorname{GL}\left(X_{\ell}\left(\Pi_{f}\right)\right)$. Then by a theorem of 
Shankar Sen ( [42]), there is an element $\varphi$ in $\mathcal{G}_{\ell}\left(\Pi_{f}\right)$ which has eigenvalues $\alpha, \beta, \gamma, \delta$ satisfying $\alpha=a+b-3>\beta=c>\gamma=d>\delta=0$, with respective multiplicities $m^{h}\left(\Pi_{f}\right), m^{W}\left(\Pi_{f}\right), m^{W}\left(\Pi_{f}\right), m^{h}\left(\Pi_{f}\right)$. But then (4.5) forces $m^{h}\left(\Pi_{f}\right)=m^{W}\left(\Pi_{f}\right)$, which is 1 . In other words, the holomorphic cusp form $\Pi^{h}$ is unique in our case.

\section{Proof of the last part of Theorem C}

We will now prove the part (c) (of Theorem C), under the hypothesis that $\pi$ is of $\ell$-adic Galois type for some $(\ell, S)$. Let $\rho_{\ell}$ be the associated 4 -dimensional, semisimple $\ell$-adic representation attached to $\pi$, having the same (degree 4) $L$-function outside $S$. Let us base change $\rho_{\ell}$ to $\overline{\mathbb{Q}}_{\ell}$ and work in the absolute setting. Also, there is a 4-dimensional Galois representation $\rho_{\ell}^{\prime}$ such that the semisimplification of $X_{\ell}\left(\Pi_{f}\right)$ is a multiple of $\rho_{\ell}^{\prime}$ (see (4.4), (4.5)). Then we have two continuous $\overline{\mathbb{Q}}_{\ell}$-representations of $\operatorname{Gal}(\overline{\mathbb{Q}} / \mathbb{Q})$ which satisfy, by the Tchebotarev density theorem,

$$
\Lambda^{2}\left(\rho_{\ell}\right) \simeq \Lambda^{2}\left(\rho_{\ell}^{\prime}\right)
$$

Note that $\Lambda^{2}\left(\rho_{\ell}^{\prime}\right)$ is of orthogonal similitude type, which can be seen by using the wedge pairing

$$
\Lambda^{2}\left(\rho_{\ell}^{\prime}\right) \times \Lambda^{2}\left(\rho_{\ell}^{\prime}\right) \rightarrow \Lambda^{4}\left(\rho_{\ell}^{\prime}\right),
$$

where the right hand side is the determinant of the 4-dimensional representation $\rho_{\ell}^{\prime}$.

One knows by Urban ( [52], Prop. 3.5), that since $m^{W}\left(\Pi_{f}\right)$ is odd, the representation $\rho_{\ell}^{\prime}$ is of symplectic type, i.e., its exterior square representation has an invariant line. In fact, this can also be deduced by using Remark 4.8, which shows that $X_{\ell}\left(\Pi_{f}\right)^{\mathrm{ss}}=\rho_{\ell}^{\prime}$, and the symplectic pairing comes from the cup product on the intersection cohomology in the middle dimension, which is alternating because the dimension is odd.

Anyhow, since the weight $w$ of $\rho_{\ell}^{\prime}$ is $w:=a+b-3$, its polarization is the $w$-th power of the cyclotomic character $\chi_{\mathrm{cyc}, \ell}$. We get a decomposition

$$
\Lambda^{2}\left(\rho_{\ell}^{\prime}\right) \simeq r\left(\rho_{\ell}^{\prime}\right) \oplus \chi_{\mathrm{cyc}, \ell}^{w},
$$

where $r=r_{5}$ is the 5-dimensional representation of $\mathrm{GSp}(4)$, taking values in $\mathrm{SGO}(5)$, which is the connected component of $\mathrm{GO}(5)$, the orthogonal similitude group; see [36], Section 1 , for a discussion of $\operatorname{SGO}(n)$.

Thanks to (5.1), $\rho_{\ell}$ is also of symplectic type, and we get a decomposition of $\Lambda^{2}\left(\rho_{\ell}\right)$, leading to an isomorphism

$$
r\left(\rho_{\ell}^{\prime}\right) \simeq r\left(\rho_{\ell}\right) .
$$

Now consider the short exact sequence of trivial $\operatorname{Gal}(\overline{\mathbb{Q}} / \mathbb{Q})$-modules

$$
1 \rightarrow \overline{\mathbb{Q}}_{\ell}^{*} \rightarrow \operatorname{GSp}\left(4, \overline{\mathbb{Q}}_{\ell}\right) \rightarrow \operatorname{SGO}\left(5, \overline{\mathbb{Q}}_{\ell}\right) \rightarrow 1 .
$$

The associated long exact cohomology sequence of $\mathcal{G}_{\mathbb{Q}}=\operatorname{Gal}(\overline{\mathbb{Q}} / \mathbb{Q})$ gives

$$
\operatorname{Hom}\left(\mathcal{G}_{\mathbb{Q}}, \overline{\mathbb{Q}}_{\ell}^{*}\right) \rightarrow \operatorname{Hom}\left(\mathcal{G}_{\mathbb{Q}}, \operatorname{GSp}\left(4, \overline{\mathbb{Q}}_{\ell}\right)\right) \rightarrow \operatorname{Hom}\left(\mathcal{G}_{\mathbb{Q}}, \operatorname{SGO}\left(5, \overline{\mathbb{Q}}_{\ell}\right)\right) \rightarrow H^{2}\left(\mathcal{G}_{\mathbb{Q}}, \overline{\mathbb{Q}}_{\ell}^{*}\right),
$$


where the last group on the right is zero by a theorem of Tate ( [43]). In any case, two Galois representations into $\operatorname{GSp}\left(4, \overline{\mathbb{Q}}_{\ell}\right)$ having the same image in $\operatorname{SGO}\left(5, \overline{\mathbb{Q}}_{\ell}\right)$, such as our $\rho_{\ell}$ and $\rho_{\ell}^{\prime}$, must satisfy

$$
\rho_{\ell}^{\prime} \simeq \rho_{\ell} \otimes \nu_{\ell}
$$

where $\nu_{\ell}$ is a 1 -dimensional $\overline{\mathbb{Q}}_{\ell}$-representation of $\mathcal{G}_{\mathbb{Q}}$. Then by $(5.1), \Lambda^{2}\left(\rho_{\ell}^{\prime}\right)$ admits a non-trivial self-twist by $\nu_{\ell}^{2}$. Taking determinants, this implies that $\nu_{\ell}^{12}=1$. So $\nu_{\ell}$ is of finite order and corresponds to a Dirichlet character $\nu$. Put

$$
\Pi_{0}^{h}:=\Pi^{h} \otimes \nu^{-1} \text {. }
$$

Now replace $\Pi^{h}$ by $\Pi_{0}^{h}$, we get

$$
L^{S}\left(s, \Pi^{h} ; r_{4}\right)=L^{S}(s, \pi) .
$$

It remains to check that (5.9) holds also at the places in $S$. One knows that for any $v \in S$, the local factor $L\left(s, \pi_{v}\right)$ (resp. $L\left(1-s, \pi_{v}^{\vee}\right)$ ) has no pole in $\Re(s) \geq 1 / 2$ (resp. in $\Re(s) \leq 1 / 2)$ (see for instance [4]). We claim that the same holds for $L\left(s, \Pi_{v}^{h} ; r_{4}\right)$ (resp. $\left.L\left(1-s, \Pi_{v}^{h^{\vee}} ; r_{4}\right)\right)$. Indeed, if $\Pi_{v}^{h}$ is tempered, as it is for $v=\infty$, there is nothing to prove. So we may assume that $v$ is finite and that $\Pi_{v}^{h}=\Pi_{v}$ is not supercuspidal. The two maximal parabolic subgroups of $\mathrm{GSp}(4)$ are both isomorphic to $\mathrm{GL}(2) \times \mathrm{GL}(1)$, and by the knowledge of the local Langlands correspondence for GL(2) (and GL(1)), one sees that the local Langlands correspondence also holds for non-supercuspidal representations of $\mathrm{GSp}(4)$. (One also has this for supercuspidal representations thanks to [25], but we will not need it here.) Let $\sigma_{v}$ be the 4-dimensional representation of the Weil-Deligne group $W_{\mathbb{Q}_{v}}^{\prime}=W_{\mathbb{Q}_{v}} \times \operatorname{SL}(2, \mathbb{C})$ attached to $\Pi_{v}$. Since $\Pi_{v}$ is not supercuspidal, and can moreover be taken to be not square-integrable, $\sigma_{v}$ is reducible, and can be written as a sum of two 2-dimensional representations $\tau_{1, v}, \tau_{2, v}$ of trivial determinant. On the other hand, we know that $\Lambda^{2}\left(\sigma_{v}\right)$ corresponds to $\Lambda^{2}\left(\pi_{v}\right)$, and so if any one-dimensional representation $\nu$ of $W_{\mathbb{Q}_{v}}^{\prime}$, which necessarily factors through $\mathbb{Q}_{v}^{*}$, occurs in $\Lambda^{2}\left(\sigma_{v}\right)=\tau_{1, v} \otimes \tau_{2, v} \oplus 1 \oplus 1$, its norm $|\nu|$ is $<1 / 2$, as $\Lambda^{2}(\pi)$ is (cf. [27]) an isobaric automorphic representation of $\mathrm{GL}(6, \mathbb{A})$. The claim now follows by considering different possibilities for $\tau_{1, v}, \tau_{2, v}$. Consequently, the poles of $L\left(s, \Pi_{v}^{h} ; r_{4}\right)$ (resp. $\left.L\left(s, \pi_{v}\right)\right)$ are disjoint from those of $L\left(1-s, \Pi_{v}^{h^{\vee}} ; r_{4}\right)\left(\right.$ resp. $L\left(1-s, \pi_{v}^{\vee}\right)$ ). For any subset $S_{1}$ of $S$ consisting of finite primes, choose a character $\nu=\nu^{S_{1}}$ which is highly ramified at $S_{1}$ and trivial at $S-S_{1}$. Now we may appeal to [46] to get all the resulting local factors at the places in $S_{1}$ to be 1 , without changing the ones at $S-S_{1}$. Arguing as in [36], proof of Prop.4.1, we get the equality $L\left(s, \pi_{v}\right)=L\left(s, \Pi_{v}^{h} ; r_{4}\right)$ at every $v \in S$.

This finishes the proof of part (c) of Theorem C.

\section{Concluding Remarks}

Suppose $\pi, \pi^{\prime}$ are cuspidal automorphic representations of $\mathrm{GL}(4, \mathbb{A})$ whose exterior square $L$-functions agree almost everywhere. It is natural to ask if they must differ by a character twist. Knowing this a priori will help remove the hypothesis in part (c) of Theorem $\mathrm{C}$ that $\pi$ is of $\ell$-adic Galois type, the reason being that we can use 
the work of the second author with M. Asgari [3] to transfer $\Pi$ back to a cuspidal automorphic representation $\pi^{\prime}$ of $\mathrm{GL}(4, \mathbb{A})$, and by construction, $\pi$ and $\pi^{\prime}$ will have the same exterior square $L$-function.

When $\pi, \pi^{\prime}$ are of symplectic type (as in the case of Theorem C), an affirmative answer should follow from a knowledge of multiplicity one for the space of cusp forms on $\operatorname{Sp}(4, \mathbb{A})$. This is analogous to the situation with $\operatorname{SL}(2)$ as in the work of the first author. To elaborate, one knows multiplicity one for SL(2) ( [35]), which implies that two cusp forms $\eta, \eta^{\prime}$ on $\mathrm{GL}(2)$ are twist-equivalent if their adjoint $L$-functions are the same (outside a finite number of factors). Analogously, in our present case of interest, there should, by functoriality, be cuspidal automorphic representations $\Pi, \Pi^{\prime}$ of $\operatorname{GSp}(4, \mathbb{A})$ whose degree $4 L$-functions agree with those of $\pi, \pi^{\prime}$ respectively. The hypothesis on the coincidence of the exterior square $L$-functions of $\pi, \pi^{\prime}$ implies that $L\left(s, \Pi ; r_{5}\right)=L\left(s, \Pi^{\prime} ; r_{5}\right)$. This should translate, by a study of the stable trace formula of $\mathrm{Sp}(4)$, analogous to that of $\mathrm{SL}(2)$ carried out by Labesse and Langlands ( [30]), to the statement that multiplicity one for $\mathrm{Sp}(4)$ would imply the twist equivalence of $\Pi, \Pi^{\prime}$. Then by the strong multiplicity one theorem, the same would hold for $\pi, \pi^{\prime}$.

In Theorem B, the weight of the anti-cyclotomic Hecke character $\chi$ is assumed to be different from 0 . In this (excluded) case, one can proceed as in this article, but $\Pi_{\infty}$ will no longer be in the discrete series, as the parameter of $\pi$ will not be regular at infinity. It is algebraic and semi-regular, however. It is likely that $\Pi_{\infty}$ should be in a (non-degenerate) limits of discrete series, the reason being that the polarization of the associated 4-dimensional, symplectic representation of $\mathrm{Gal}(\overline{\mathbb{Q}} / \mathbb{Q})$ is the transfer of $\chi$ to $\mathbb{Q}$ times the cyclotomic character, which is odd. Still, the existence of a global holomorphic $L$-equivalent $\Pi^{h}$ is not clear, as one cannot appeal to the $\ell$-adic cohomology of the Siegel threefold. However, such a $\Pi^{h}$ should exist, as predicted by the conjectures of J. Arthur, and this should follow from a thorough understanding of the comparison of the stable trace formula for $\mathrm{GSp}(4)$ and the twisted, stable trace formula for GL(4) relative to the automorphism $g \rightarrow{ }^{t} g^{-1}$. This appears to be close given the works of Arthur [1] on this comparison, and of D. Whitehouse ( [54]), who recently proved the crucially needed twisted, weighted fundamental lemma conjectured by Arthur.

\section{Acknowledgements}

We would like to acknowledge helpful conversations with J. Cogdell and D. Soudry, and thank A. Wiles for his question regarding Theorem B, which started this line of investigation. The first author thanks J. Tilouine for the reference to the needed papers of Laumon and Weissauer (on the cohomology of Siegel modular threefolds), which have quite recently appeared in an Astérisque volume. We also thank the referee for reading the paper carefully and for making good suggestions, which have led to an improvement of the presentation. Finally, we would like to acknowledge support from the NSF for this work through our individual research grants DMS-0402044 (D.R.) and DMS-0200325 (F.S.). 


\section{References}

[1] J. Arthur, Automorphic representations of GSp(4), Contributions to Automorphic forms, Geometry, and Number Theory (2004) 65-81.

[2] J. Arthur and L. Clozel, Simple Algebras, Base Change and the Advanced Theory of the Trace Formula, Vol. 120 of Ann. Math. Studies (1989).

[3] M. Asgari and F. Shahidi, Generic transfer for general spin groups, Duke Math Journal 132 (2006) 137-190.

[4] L. Barthel and D. D.Ramakrishnan, A nonvanishing result for twists of L-functions of GL( $n)$, Duke Math Journal 74 (1994), no. 3, 681-700.

[5] A. A. Beilinson, J. Bernstein, and P. Deligne, Faisceaux pervers, Astérisque 100 (1982) 5-171.

[6] C. Breuil, B. Conrad, F. Diamond, and R. Taylor, On the modularity of elliptic curves over $\mathbf{Q}$ : wild 3-adic exercises, J. Amer. Math. Soc. 14 (2001), no. 4, 843-939.

[7] D. Bump and S. Friedberg, The exterior square automorphic L-functions on GL( $n)$, in Festschrift in honor of I. I. Piatetski-Shapiro on the occasion of his sixtieth birthday, Part II (Ramat Aviv, 1989), Vol. 3 of Israel Math. Conf. Proc., 47-65, Weizmann, Jerusalem (1990).

[8] D. Bump and D. Ginzburg, Symmetric square L-functions on GL( $r)$, Annals of Math.

[9] H. Carayol, Sur les représentations $\ell$-adiques associées aux formes modulaires de Hilbert, Ann. Sci. École Norm. Sup.

[10] L. Clozel, Motifs et formes automorphes, Perspectives in Math 10 (1990) 77-159.

[11] - Représentations galoisiennes associées aux représentations automorphes autoduales de GL(n), Publ. Math. IHES 73 (1991) 97-145.

[12] J. W. Cogdell, H. H. Kim, I. I. Piatetski-Shapiro, and F. Shahidi, Functoriality for the classical groups, Publ. Math. Inst. Hautes Études Sci. (2004), no. 99, 163-233.

[13] P. Deligne, Formes modulaires et reprsentations $\ell$-adiques, Séminaire Bourbaki 1968/69 355 (1971) 139-172.

[14] G. Faltings, Crystalline cohomology and p-adic Galois-representations, in Algebraic analysis, geometry, and number theory (Baltimore, MD, 1988), 25-80, Johns Hopkins Univ. Press, Baltimore, MD (1989).

[15] D. Ginzburg, S. Rallis, and D. Soudry, On explicit lifts of cusp forms from GL $_{m}$ to classical groups, Ann. of Math. (2) 150 (1999), no. 3, 807-866.

[16] M. Harris, D. Soudry, and R. Taylor, l-adic representations associated to modular forms over imaginary quadratic fields. I. Lifting to $\mathrm{GSp}_{4}(\mathbf{Q})$, Invent. Math. 112 (1993), no. 2, 377-411.

[17] M. Harris and R. Taylor, The geometry and cohomology of some simple Shimura varieties, Vol 151 of Annals of Mathematics Studies, Princeton University Press, Princeton, NJ (2001). With an appendix by Vladimir G. Berkovich.

[18] G. Henniart, Une preuve simple des conjectures de Langlands pour $\mathrm{GL}(n)$ sur un corps p-adique, Invent. Math. 139 (2000), no. 2, 439-455.

[19] G. Henniart and R. Herb, Automorphic induction for GL $(n)$ (over local non-Archimedean fields), Duke Math. J. 78 (1995), no. 1, 131-192.

[20] T. Ibukiyama, Numerical Example of a Siegel modular form having the cubic zeta function, Preprint.

[21] H. Jacquet, I. I. Piatetski-Shapiro, and J. Shalika, Conducteur des représentations du groupe linéaire, Math. Ann. 256 (1981), no. 2, 199-214.

[22] H. Jacquet, I. I. Piatetskii-Shapiro, and J. A. Shalika, Rankin-Selberg convolutions, Amer. J. Math. 105 (1983), no. 2, 367-464.

[23] H. Jacquet and J. Shalika, Exterior square L-functions, in Automorphic forms, Shimura varieties, and L-functions, Vol. II (Ann Arbor, MI, 1988), Vol. 11 of Perspect. Math., 143-226, Academic Press, Boston, MA (1990).

[24] H. Jacquet and J. A. Shalika, On Euler products and the classification of automorphic forms I, II, Amer. J. Math. 103 (1981), no. 3, 4, 499-558, 777-815.

[25] D. Jiang and D. Soudry, Generic representations and local Langlands reciprocity law for p-adic $\mathrm{SO}_{2 n+1}$, in Contributions to automorphic forms, geometry, and number theory, 457-519, Johns Hopkins Univ. Press, Baltimore, MD (2004).

[26] - On the multiplicity one theorem for generic automorphic forms on GSp(4) (Preprint (2006), Pacific Journal of Math., to appear) 
[27] H. H. Kim, Functoriality for the exterior square of $\mathrm{GL}_{4}$ and the symmetric fourth of $\mathrm{GL}_{2}$, J. Amer. Math. Soc. 16 (2003), no. 1, 139-183. With appendix 1 by Dinakar Ramakrishnan and appendix 2 by Kim and Peter Sarnak.

[28] H. H. Kim and F. Shahidi, Cuspidality of symmetric powers with applications, Duke Math. J. 112 (2002), no. $1,177-197$.

[29] - Functorial products for $\mathrm{GL}_{2} \times \mathrm{GL}_{3}$ and the symmetric cube for $\mathrm{GL}_{2}$, Ann. of Math. (2) 155 (2002), no. 3, 837-893. With an appendix by Colin J. Bushnell and Guy Henniart.

[30] J.-P. Labesse and R. P. Langlands, L-indistinguishability for SL(2), Canad. J. Math. 31 (1979), no. $4,726-785$

[31] G. Laumon, Sur la cohomologie à supports compacts des variétés de Shimura pour $\mathrm{GSp}(4)_{\mathbf{Q}}$, Compositio Math. 105 (1997), no. 3, 267-359.

[32] _ Fonctions zêtas des variétés de Siegel de dimension trois, Astérisque (2005), no. 302, 1-66. Formes automorphes. II. Le cas du groupe GSp(4).

[33] E. Looijenga, $L^{2}$-cohomology of locally symmetric varieties, Compositio Math. 67 (1988), no. 1, $3-20$.

[34] K. Paranjape and D. Ramakrishnan, Modular forms and Calabi-Yau varieties, In preparation.

[35] D. Ramakrishnan, Modularity of the Rankin-Selberg L-series, and multiplicity one for SL(2), Ann. of Math. (2) 152 (2000), no. 1, 45-111.

[36] - Modularity of solvable Artin representations of GO(4)-type, Int. Math. Res. Not. (2002), no. $1,1-54$.

[37] - Irreducibility and Cuspidality, in Representation Theory and Automorphic Forms, 1-36. Progress in Mathematics, Birkhüser, Boston (2006).

[38] K. A. Ribet, Galois representations attached to eigenforms with Nebentypus, in Modular functions of one variable, V (Proc. Second Internat. Conf., Univ. Bonn, Bonn, 1976), 17-51. Lecture Notes in Math., Vol. 601, Springer, Berlin (1977).

[39] B. Roberts and R. Schmidt, On modular forms for the paramodular groups, in Automorphic forms and zeta functions, 334-364, World Sci. Publ., Hackensack, NJ (2006).

[40] L. Saper and M. Stern, $L_{2}$-cohomology of arithmetic varieties, Ann. of Math. (2) 132 (1990), no. $1,1-69$.

[41] R. Schmidt, Iwahori-spherical representations of GSp(4) and Siegel modular forms of degree 2 with square-free level, J. Math. Soc. Japan 57 (2005), no. 1, 259-293.

[42] S. Sen, Lie algebras of Galois groups arising from Hodge-Tate modules, Ann. of Math. (2) 97 (1973) 160-170.

[43] J.-P. Serre, Modular forms of weight one and Galois representations, in Algebraic number fields: $L$-functions and Galois properties (Proc. Sympos., Univ. Durham, Durham, 1975), 193-268, Academic Press, London (1977).

[44] _ Abelian $l$-adic representations and elliptic curves, Vol. 7 of Research Notes in Mathematics, A K Peters Ltd., Wellesley, MA (1998). With the collaboration of Willem Kuyk and John Labute, Revised reprint of the 1968 original.

[45] F. Shahidi, On certain L-functions, Amer. J. Math. 103 (1981), no. 2, 297-355.

[46] - Twists of a general class of L-functions by highly ramified characters, Canad. Math. Bull. 43 (2000), no. 3, 380-384.

[47] — Infinite dimensional groups and automorphic L-functions, Pure Appl. Math. Q. 1 (2005), no. 3, 683-699.

[48] D. Soudry, On Langlands functoriality from classical groups to $\mathrm{GL}_{n}$, Astérisque (2005), no. 298, 335-390. Automorphic forms. I.

[49] R. Taylor, On the l-adic cohomology of Siegel threefolds, Invent. Math. 114 (1993), no. 2, 289-310.

[50] R. Taylor and A. Wiles, Ring-theoretic properties of certain Hecke algebras, Ann. of Math. (2) 141 (1995), no. 3, 553-572.

[51] T. Tsuji, p-adic étale cohomology and crystalline cohomology in the semi-stable reduction case, Invent. Math. 137 (1999), no. 2, 233-411.

[52] E. Urban, Selmer groups and the Eisenstein-Klingen ideal, Duke Math. J. 106 (2001), no. 3, $485-525$.

[53] R. Weissauer, Four dimensional Galois representations, Astérisque (2005), no. 302, 67-150. Formes automorphes. II. Le cas du groupe GSp(4). 
[54] D. Whitehouse, The twisted weighted fundamental lemma for the transfer of automorphic forms from GSp(4), Astérisque (2005), no. 302, 291-436. Formes automorphes. II. Le cas du groupe $\operatorname{GSp}(4)$.

[55] A. Wiles, Modular elliptic curves and Fermat's last theorem, Ann. of Math. (2) 141 (1995), no. $3,443-551$.

Department of Mathematicss, California Institute of Technology, Pasadena, CA 91125

E-mail address: dinakar@caltech.edu

Department of Mathematics, Purdue University, West Lafayette, IN 47907

E-mail address: shahidi@math.purdue.edu 